\title{
Identification of the unknown boundary condition in a linear parabolic equation
}

\section{Ali Demir ${ }^{1}$ and Ebru Ozbilge 2* $^{*}$}

${ }^{\text {"Correspondence: }}$

ebru.ozbilge@ieu.edu.tr

${ }^{2}$ Department of Mathematics,

Faculty of Science and Literature,

Izmir University of Economics,

Sakarya Caddesi, No.156, Balcova,

Izmir, 35330, Turkey

Full list of author information is

available at the end of the article

\section{Abstract}

In this article, a semigroup approach is presented for the mathematical analysis of the inverse problems of identifying the unknown boundary condition $u(1, t)=f(t)$ in a linear parabolic equation $u_{t}(x, t)=\left(k(u(x, t)) u_{x}(x, t)\right)_{x}$ with Dirichlet boundary conditions $u(0, t)=\psi_{0}, u(1, t)=f(t)$ by making use of the over measured data $u\left(x_{0}, t\right)=\psi_{1}$ and $u_{x}\left(x_{0}, t\right)=\psi_{2}$ separately.

\section{Introduction}

Consider the following initial boundary value problem for the linear diffusion equation:

$$
\begin{aligned}
& u_{t}(x, t)=\left(k(x) u_{x}(x, t)\right)_{x}, \quad(x, t) \in \Omega_{T}, \\
& u(x, 0)=g(x), \quad 0<x<1, \\
& u(0, t)=\psi_{0}, \quad u(1, t)=f(t), \quad 0<t<T,
\end{aligned}
$$

where $\Omega_{T}=\left\{(x, t) \in R^{2}: 0<x<1,0<t \leq T\right\}$. The left boundary value $\psi_{0}$ is assumed to be constant. The functions $c_{1}>k(x) \geq c_{0}>0$ and $g(x)$ satisfy the following conditions:

(C1) $k(x) \in H^{1,2}[0,1]$;

(C2) $g(x) \in H^{2,2}[0,1], g(0)=\psi_{0}, g(1)=f(0)$.

The initial boundary value problem (1) has the unique solution $u(x, t)$ satisfying $u(x, t) \in$ $H^{2,2}[0,1] \cap H^{1,2}[0,1][1-4]$ under these conditions.

In physics, many applications of this problem can be found. The simple model of flame propagation and the spread of a biological populations, where $u=u(x, t), k(x)$ denote the temperature and density respectively, are given by the equation in problem (1). Especially $k=k(x)$ represents the density-dependent coefficient in the problems of the spread of biological populations [5-9].

Let us consider the inverse problems [10] of determining boundary $u(1, t)$ at $x=1$ in problem (1) from Dirichlet type of measured output data at the boundaries $x=x_{0}$ :

$$
u\left(x_{0}, t\right)=\psi_{1}, \quad t \in(0, T]
$$

and from Neumann type of measured output data at the boundaries $x=x_{0}$ :

$$
u_{x}\left(x_{0}, t\right)=\psi_{2}, \quad t \in(0, T]
$$

(0) 2013 Demir and Ozbilge; licensee Springer. This is an Open Access article distributed under the terms of the Creative Commons Attribution License (http://creativecommons.org/licenses/by/2.0), which permits unrestricted use, distribution, and reproduction in any medium, provided the original work is properly cited. 
Here the solution of the parabolic problem (1) is denoted by $u=u(x, t)$. In this context, the parabolic problem (1) will be referred to as a direct (forward) problem with the inputs $g(x), k(x)$ and $f(t)$. Notice that $u\left(x_{0}, 0\right)=\psi_{1}$ and $u_{x}\left(x_{0}, 0\right)=\psi_{2}$. Therefore it is assumed that the functions $u\left(x_{0}, t\right)=\psi_{1}$ and $u_{x}\left(x_{0}, t\right)=\psi_{2}$ respectively satisfy the consistency conditions $\psi_{1}=g\left(x_{0}\right)$ and $\psi_{2}=g^{\prime}\left(x_{0}\right)$.

The purpose of this paper is to determine the boundary function $u(1, t)$ at $x=1$ via the semigroup approach which is studied by [11-15].

The paper is organized as follows. In Section 2, an analysis of the semigroup approach is given for the inverse problem with the single measured output data $u\left(x_{0}, t\right)=\psi_{1}$ given at $x=x_{0}$. A similar analysis is applied to the inverse problem with the single measured output data $u_{x}\left(x_{0}, t\right)=\psi_{2}$ given at the point $x=x_{0}$ in Section 3 . Some concluding remarks are given in Section 4.

\section{Analysis of the inverse problem of the boundary condition by Dirichlet type of over measured data $u\left(x_{0}, t\right)=\psi_{1}$}

Consider now the inverse problem with one measured output data $u\left(x_{0}, t\right)=\psi_{1}$ at $x=x_{0}$. In order to formulate the solution of the parabolic problem (1) in terms of semigroup, let us first arrange the parabolic equation as follows:

$$
u_{t}(x, t)-\left(k(0) u_{x}(x, t)\right)_{x}=\left([k(x)-k(0)] u_{x}(x, t)\right)_{x}, \quad(x, t) \in \Omega_{T} .
$$

Then the initial boundary value problem (1) can be rewritten in the following form:

$$
\begin{aligned}
& u_{t}(x, t)-k(0) u_{x x}(x, t)=\left((k(x)-k(0)) u_{x}(x, t)\right)_{x}, \quad(x, t) \in \Omega_{T}, \\
& u(x, 0)=g(x), \quad 0<x<1, \\
& u(0, t)=\psi_{0}, \quad u(1, t)=f(t), \quad 0<t<T .
\end{aligned}
$$

In order to determine the unknown boundary condition $u(1, t)=f(t)$, we need to determine the solution of the following parabolic problem:

$$
\begin{aligned}
& u_{t}(x, t)-k(0) u_{x x}(x, t)=\left((k(x)-k(0)) u_{x}(x, t)\right)_{x}, \quad(x, t) \in \Omega_{T}, \\
& u(x, 0)=g(x), \quad 0<x<1, \\
& u(0, t)=\psi_{0}, \quad u\left(x_{0}, t\right)=\psi_{1}, \quad 0<t<T .
\end{aligned}
$$

To formulate the solution of the above problem in terms of semigroup, we need to define a new function

$$
v(x, t)=u(x, t)+\frac{\left(\psi_{0}-\psi_{1}\right) x}{x_{0}}-\psi_{0}, \quad x \in[0,1],
$$

which satisfies the following parabolic problem:

$$
\begin{aligned}
& v_{t}(x, t)+A[v(x, t)]=\left((k(x)-k(0))\left(v_{x}(x, t)-\frac{\left(\psi_{0}-\psi_{1}\right)}{x_{0}}\right)\right)_{x}, \quad(x, t) \in \Omega_{T}, \\
& v(x, 0)=g(x)+\frac{\left(\psi_{0}-\psi_{1}\right) x}{x_{0}}-\psi_{0}, \quad 0<x<1, \\
& v(0, t)=0, \quad v\left(x_{0}, t\right)=0, \quad 0<t<T .
\end{aligned}
$$


Here, $A[\cdot]:=-k(0) \frac{d^{2}[\cdot]}{d x^{2}}$ is a second-order differential operator and its domain is $D_{A}=\{v \in$ $\left.H^{2,2}\left(0, x_{0}\right) \cap H^{1,2}\left[0, x_{0}\right]: v(0)=v\left(x_{0}\right)=0\right\}$, where $H_{0}^{2,2}\left(0, x_{0}\right)=\overline{C_{0}^{2}\left(0, x_{0}\right)}$ and $H_{0}^{1,2}\left[0, x_{0}\right]=$ $\overline{C_{0}^{1}\left[0, x_{0}\right]}$ are Sobolev spaces. Obviously, by completion $g(x) \in D_{A}$ since the initial value function $g(x)$ belongs to $C^{3}[0,1]$. Hence $D_{A}$ is dense in $H_{0}^{2,2}[0,1]$, which is a necessary condition for being an infinitesimal generator.

In the following, despite doing the calculations in the smooth function space, by completion they are valid in the Sobolev space.

Let us denote a semigroup of linear operators by $T(t)$ generated by the operator $-A$ $[9,10]$. We can easily find the eigenvalues and eigenfunctions of the differential operator $A$. Moreover, the semigroup $T(t)$ can be easily constructed by using the eigenvalues and eigenfunctions of the infinitesimal generator $A$. Hence we first consider the following eigenvalue problem:

$$
\begin{aligned}
& A \phi(x)=\lambda \phi(x), \\
& \phi(0)=0 ; \quad \phi\left(x_{0}\right)=0 .
\end{aligned}
$$

It can easily be determined that the eigenvalues are $\lambda_{n}=k(0) \frac{n^{2} \pi^{2}}{x_{0}^{2}}$ for all $n=0,1, \ldots$ and hence the corresponding eigenfunctions are $\phi_{n}(x)=\sin \left(\frac{n \pi x}{x_{0}}\right)$. This allows us to represent the semigroup $T(t)$ in the following way:

$$
T(t) U(x, s)=\sum_{n=0}^{\infty}\left\langle\phi_{n}(x), U(x, s)\right| e^{-\lambda_{n} t} \phi_{n}(x),
$$

where $\left\langle\phi_{n}(x), U(x, s)\right\rangle=\int_{0}^{1} \phi_{n}(x) U(x, s) d x$. Under this representation, the null space of the semigroup $T(t)$ of the linear operators can be defined as follows:

$$
N(T)=\left\{U(x, s):\left\langle\phi_{n}(x), U(x, s)\right\rangle=0, \text { for all } n=0,1,2,3, \ldots\right\}
$$

From the construction of the semigroup $T(t)$, it can be concluded that the null space of it consists of only zero function, i.e., $N(T)=\{0\}$. This result implies the uniqueness of the unknown boundary condition $u(1, t)$.

The unique solution of the initial-boundary value problem (7) in terms of semigroup $T(t)$ can be represented in the following form:

$$
v(x, t)=T(t) v(x, 0)+\int_{0}^{t} T(t-s)\left((k(x)-k(0))\left(v_{x}(x, s)-\frac{\left(\psi_{0}-\psi_{1}\right)}{x_{0}}\right)\right)_{x} d s .
$$

Now, by using the identity (6) and taking the initial value $u(x, 0)=g(x)$ into account, the integral equation for the solution $u(x, t)$ of the parabolic problem (5) in terms of semigroup can be written in the following form:

$$
\begin{aligned}
u(x, t)= & \psi_{0}-\frac{\left(\psi_{0}-\psi_{1}\right) x}{x_{0}}+T(t)\left(g(x)+\frac{\left(\psi_{0}-\psi_{1}\right) x}{x_{0}}-\psi_{0}\right) \\
& +\int_{0}^{t} T(t-s)\left((k(x)-k(0)) u_{x}(x, s)\right)_{x} d s .
\end{aligned}
$$


In order to arrange the above integral equation, let us define the following:

$$
\begin{aligned}
& \zeta(x)=\left(g(x)+\frac{\left(\psi_{0}-\psi_{1}\right) x}{x_{0}}-\psi_{0}\right), \\
& \xi(x, t)=\left((k(x)-k(0)) u_{x}(x, t)\right)_{x} .
\end{aligned}
$$

Then we can rewrite the integral equation in terms of $\zeta(x)$ and $\xi(x, s)$ in the following form:

$$
u(x, t)=\psi_{0}-\frac{\left(\psi_{0}-\psi_{1}\right) x}{x_{0}}+(T(t) \zeta(\cdot))(x, t)+\int_{0}^{t}(T(t-s) \xi(\cdot, s))(x, t, s) d s
$$

This is the integral representation of a solution of the initial-boundary value problem (5) on $\Omega_{T}=\left\{(x, t) \in R^{2}: 0<x<x_{0}, 0<t \leq T\right\}$. It is obvious from the eigenfunctions $\phi_{n}(x)$, the domain of eigenfunctions can be extended to a closed interval $[0,1]$. Moreover, they are continuous on $[0,1]$. Under this extension, the uniqueness of the initialboundary value problems (4) and (5) imply that the integral representation (11) becomes the integral representation of a solution of the initial-boundary value problem (4) on $\Omega_{T}=\left\{(x, t) \in R^{2}: 0<x<1,0<t \leq T\right\}$.

At this stage, it is obvious that the solution of the inverse problem can easily be obtained by substituting $x=1$ into the integral representation (11) of the solution $u(x, t)$ :

$$
u(1, t)=f(t)=\psi_{0}-\frac{\left(\psi_{0}-\psi_{1}\right)}{x_{0}}+(T(t) \zeta(\cdot))(1, t)+\int_{0}^{t}(T(t-s) \xi(\cdot, s))(1, t, s) d s,
$$

which implies that $f(t)$ can be determined analytically. The right-hand side of identity (12) defines the semigroup representation of the unknown boundary condition $u(1, t)$ at $x=1$.

Substituting $t=0$ into equation (12) yields

$$
u(1,0)=f(0)=g(1)
$$

which is the condition we have for the initial-boundary value problems (1). Similarly, plugging $t=0$ into equation (11) produces the following:

$$
u(x, 0)=g(x)
$$

which is the initial condition we have.

\section{Analysis of the inverse problem of the boundary condition by Neumann type of over measured data $u_{x}\left(x_{0}, t\right)=\psi_{2}$}

Consider now the inverse problem with one measured output data $u_{x}\left(x_{0}, t\right)=\psi_{2}$ at $x=x_{0}$. In order to formulate the solution of the parabolic problem (1) in terms of semigroup, as before, we arrange the parabolic equation as follows:

$$
u_{t}(x, t)-\left(k(0) u_{x}(x, t)\right)_{x}=\left([k(x)-k(0)] u_{x}(x, t)\right)_{x^{\prime}} \quad(x, t) \in \Omega_{T} .
$$


Then the initial boundary value problem (1) can be rewritten in the following form:

$$
\begin{aligned}
& u_{t}(x, t)-k(0) u_{x x}(x, t)=\left((k(x)-k(0)) u_{x}(x, t)\right)_{x}, \quad(x, t) \in \Omega_{T}, \\
& u(x, 0)=g(x), \quad 0<x<1, \\
& u(0, t)=\psi_{0}, \quad u(1, t)=f(t), \quad 0<t<T .
\end{aligned}
$$

In order to determine the unknown boundary condition $u(1, t)=f(t)$, we need to determine the solution of the following parabolic problem:

$$
\begin{aligned}
& u_{t}(x, t)-k(0) u_{x x}(x, t)=\left((k(x)-k(0)) u_{x}(x, t)\right)_{x}, \quad(x, t) \in \Omega_{T}, \\
& u(x, 0)=g(x), \quad 0<x<1, \\
& u(0, t)=\psi_{0}, \quad u_{x}\left(x_{0}, t\right)=\psi_{2}, \quad 0<t<T .
\end{aligned}
$$

To formulate the solution of the above problem in terms of semigroup, we need to define a new function

$$
v(x, t)=u(x, t)-\psi_{2} x-\psi_{0}, \quad x \in[0,1]
$$

which satisfies the following parabolic problem:

$$
\begin{aligned}
& v_{t}(x, t)+B[v(x, t)]=\left((k(x)-k(0))\left(v_{x}(x, t)+\psi_{2}\right)\right)_{x}, \quad(x, t) \in \Omega_{T}, \\
& v(x, 0)=g(x)-\psi_{2} x-\psi_{0}, \quad 0<x<1 \\
& v(0, t)=0, \quad v_{x}\left(x_{0}, t\right)=0, \quad 0<t<T .
\end{aligned}
$$

Here $B[\cdot]:=-k(0) \frac{d^{2}[\cdot]}{d x^{2}}$ is a second-order differential operator, its domain is $D_{B}=\{v \in$ $\left.H^{2,2}\left(0, x_{0}\right) \cap H^{1,2}\left[0, x_{0}\right]: v(0)=v_{x}\left(x_{0}\right)=0\right\}$. It is clear from the definition of $D_{B}$ that $D_{B} \subset$ $H^{2,2}\left[0, x_{0}\right]$.

Let us denote the semigroup of linear operators by $S(t)$ generated by the operator $-B$ $[9,10]$. We can easily find the eigenvalues and eigenfunctions of the differential operator $B$. Moreover, the semigroup $S(t)$ can be easily constructed by using the eigenvalues and eigenfunctions of the infinitesimal generator $B$. Hence we first consider the following eigenvalue problem:

$$
\begin{aligned}
& B \phi(x)=\lambda \phi(x), \\
& \phi(0)=0 ; \quad \phi_{x}\left(x_{0}\right)=0 .
\end{aligned}
$$

The eigenvalues are determined as $\lambda_{n}=k(0) \frac{(2 n+1)^{2} \pi^{2}}{4 x_{0}^{2}}$ for all $n=0,1, \ldots$ Therefore the corresponding eigenfunctions are $\phi_{n}(x)=\sin \left(\frac{(2 n+1) \pi x}{2 x_{0}}\right)$. Under these eigenfunctions and eigenvalues, the semigroup $S(t)$ can be represented in the following way:

$$
S(t) U(x, s)=\sum_{n=0}^{\infty}\left\langle\phi_{n}(x), U(x, s)\right\rangle e^{-\lambda_{n} t} \phi_{n}(x),
$$


where $\left\langle\phi_{n}(x), U(x, s)\right\rangle=\int_{0}^{1} \phi_{n}(x) U(x, s) d x$. Under this representation, the null space of the semigroup $S(t)$ of the linear operators can be defined as follows:

$$
N(S)=\left\{U(x, s):\left\langle\phi_{n}(x), U(x, s)\right\rangle=0 \text {, for all } n=0,1,2,3, \ldots\right\} .
$$

As in the previous section, the construction of the semigroup $S(t)$ implies that the null space of it consists of only zero function, i.e., $N(S)=\{0\}$. The uniqueness of the unknown boundary condition $u(1, t)$ follows from this result.

The unique solution of the initial-boundary value problem (16) in terms of semigroup $S(t)$ can be represented in the following form:

$$
v(x, t)=S(t) v(x, 0)+\int_{0}^{t} S(t-s)\left((k(x)-k(0))\left(v_{x}(x, s)+\psi_{2}\right)\right)_{x} d s .
$$

Now, by using the identity (17) and taking the initial value $u(x, 0)=g(x)$ into account, the integral equation for the solution $u(x, t)$ of the parabolic problem (14) in terms of semigroup can be written in the following form:

$$
\begin{aligned}
u(x, t)= & \psi_{0}+\psi_{2} x+S(t)\left(g(x)-\psi_{0}-\psi_{2} x\right) \\
& +\int_{0}^{t} S(t-s)\left((k(x)-k(0)) u_{x}(x, s)\right)_{x} d s
\end{aligned}
$$

In order to arrange the above integral equation, let us define the following:

$$
\begin{aligned}
& \zeta(x)=\left(g(x)-\psi_{0}-\psi_{2} x\right), \\
& \xi(x, t)=\left((k(x)-k(0)) u_{x}(x, t)\right)_{x} .
\end{aligned}
$$

Then we can rewrite the integral equation in terms of $\zeta(x)$ and $\xi(x, s)$ in the following form:

$$
u(x, t)=\psi_{0}+\psi_{2} x+(S(t) \zeta(\cdot))(x, t)+\int_{0}^{t}(S(t-s) \xi(\cdot, s))(x, t, s) d s
$$

This is the integral representation of a solution of the initial-boundary value problem (15) on $\Omega_{T}=\left\{(x, t) \in R^{2}: 0<x<x_{0}, 0<t \leq T\right\}$. It is obvious from the eigenfunctions $\phi_{n}(x)$, the domain of eigenfunctions can be extended to a closed interval $[0,1]$. Moreover, they are continuous on $[0,1]$. Under this extension, the uniqueness of the initialboundary value problems (15) and (16) imply that integral representation (22) becomes the integral representation of a solution of the initial-boundary value problem (16) on $\Omega_{T}=\left\{(x, t) \in R^{2}: 0<x<1,0<t \leq T\right\}$.

Substituting $x=1$ into the integral representation (22) of the solution $u(x, t)$ yields

$$
u(1, t)=f(t)=\psi_{0}+\psi_{2}+(S(t) \zeta(\cdot))(1, t)+\int_{0}^{t}(S(t-s) \xi(\cdot, s))(1, t, s) d s
$$

which implies that $f(t)$ can be determined analytically.

The right-hand side of identity (23) defines the semigroup representation of the unknown boundary condition $u(1, t)$ at $x=1$. 


\section{Conclusion}

The purpose of this study is to identify the unknown boundary condition $u(1, t)$ at $x=1$ via the semigroup approach by using the over measured data $u\left(x_{0}, t\right)=\psi_{1}$ and $u_{x}\left(x_{0}, t\right)=\psi_{2}$. The crucial point here is the unique extensions of the solutions on $\left\{(x, t) \in R^{2}: 0<x<\right.$ $\left.x_{0}, 0<t \leq T\right\}$ to $\left\{(x, t) \in R^{2}: 0<x<1,0<t \leq T\right\}$ which are implied by the uniqueness of the solutions. This key leads to the integral representation of the unknown boundary condition $u(1, t)$ at $x=1$ obtained analytically.

\section{Competing interests}

The authors declare that they have no competing interests.

\section{Authors' contributions}

Both authors contributed equally to the manuscript and read and approved the final manuscript.

\section{Author details}

${ }^{1}$ Department of Mathematics, Kocaeli University, Umuttepe, Izmit, Kocaeli 41380, Turkey. ${ }^{2}$ Department of Mathematics, Faculty of Science and Literature, Izmir University of Economics, Sakarya Caddesi, No.156, Balcova, Izmir, 35330, Turkey.

\section{Acknowledgements}

Dedicated to Professor Hari M Srivastava.

The research was supported by parts by the Scientific and Technical Research Council (TUBITAK) of Turkey and Izmir University of Economics.

\section{Received: 27 December 2012 Accepted: 18 February 2013 Published: 7 March 2013}

\section{References}

1. DuChateau, P: Monotonicity and invertibility of coefficient-to-data mappings for parabolic inverse problems. SIAM J. Math. Anal. 26, 1473-1487 (1995)

2. Isakov, V: On uniqueness in inverse problems for quasilinear parabolic equations. Arch. Ration. Mech. Anal. 124, 1-13 (1993)

3. Pilant, MS, Rundell, W: A uniqueness theorem for conductivity from overspecified boundary data. J. Math. Anal. Appl. 136, 20-28 (1988)

4. Renardy, M, Rogers, R: An Introduction to Partial Differential Equations. Springer, New York (2004)

5. Cannon, JR: The One-Dimensional Heat Equation. Addison-Wesley, Reading (1984)

6. DuChateau, P, Thelwell, R, Butters, G: Analysis of an adjoint problem approach to the identification of an unknown diffusion coefficient. Inverse Probl. 20, 601-625 (2004)

7. Showalter, R: Monotone Operators in Banach Spaces and Nonlinear Partial Differential Equations. Am. Math. Soc., Providence (1997)

8. Hasanov, A, Demir, A, Erdem, A: Monotonicity of input-output mappings in inverse coefficient and source problem for parabolic equations. J. Math. Anal. Appl. 335, 1434-1451 (2007)

9. Hasanov, A, DuChateau, P, Pektas, B: An adjoint approach and coarse-fine mesh method for identification of the diffusion coefficient in a linear parabolic equations. J. Inverse III-Posed Probl. 14, 435-463 (2006)

10. DuChateau, P, Gottlieb, J: Introduction to Inverse Problems in Partial Differential Equations for Engineers, Physicists and Mathematicians. Kluwer Academic, Dordrecht (1996)

11. Ashyralyev, A, San, ME: An approximation of semigroup method for stochastic parabolic equations. Abstr. Appl. Anal. (in press)

12. Demir, A, Ozbilge, E: Semigroup approach for identification of the unknown diffusion coefficient in a quasi-linear parabolic equation. Math. Methods Appl. Sci. 30, 1283-1294 (2007)

13. Ozbilge, E: Identification of the unknown diffusion coefficient in a quasi-linear parabolic equation by semigroup approach with mixed boundary conditions. Math. Methods Appl. Sci. 31, 1333-1344 (2008)

14. Demir, A, Ozbilge, E: Analysis of a semigroup approach in the inverse problem of identifying an unknown coefficient. Math. Methods Appl. Sci. 31, 1635-1645 (2008)

15. Demir, A, Hasanov, A: Identification of the unknown diffusion coefficient in a linear parabolic equation by the semigroup approach. J. Math. Anal. Appl. 340, 5-15 (2008)

doi:10.1186/1029-242X-2013-96

Cite this article as: Demir and Ozbilge: Identification of the unknown boundary condition in a linear parabolic equation. Journal of Inequalities and Applications 2013 2013:96. 